\title{
Study of Binary Partition Tree Pruning Techniques for Polarimetric SAR images
}

\author{
Philippe Salembier ${ }^{\star}$ \\ Technical University of Catalonia, Barcelona, Spain \\ <philippe.salembier@upc.edu>
}

\begin{abstract}
This paper investigates several pruning techniques applied on Binary Partition Trees (BPTs) and their usefulness for low-level processing of PolSAR images. BPTs group pixels to form homogeneous regions, which are hierarchically structured by inclusion in a binary tree. They provide multiple resolutions of description and easy access to subsets of regions. Once constructed, BPTs can be used for a large number of applications. Many of these applications consist in populating the tree with a specific feature and in applying a graph-cut called pruning to extract a partition of the space. In this paper, different pruning examples involving the optimization of a global criterion are discussed and analyzed in the context of PolSAR images for segmentation. Initial experiments are also reported on the use of Minkowski norms in the definition of the optimization criterion.
\end{abstract}

Keywords: Binary Partition Tree, PolSAR, graph-cut, pruning, speckle noise, segmentation.

\section{Introduction}

The application of Binary Partition Trees (BPTs) [18] for remote sensing applications such as Polarimetric SAR (PolSAR) [2] and hyperspectral images [20,21] is currently gaining interest. BPTs are hierarchical region-based representations in which pixels are grouped by similarity. Their construction is often based on an iterative region-merging algorithm: starting from an initial partition, the pair of most similar neighboring regions is iteratively merged until one region representing the entire image support is obtained. The BPT essentially stores the complete merging sequence in a binary tree structure. Once constructed, BPTs can be used for a large number of tasks including image filtering with connected operators, segmentation, object detection or classification $[18,3]$. Many of these tasks involve the extraction of a partition from the BPT through a graph cut.

In this paper, we focus on low level PolSAR image processing tasks. We study in particular the interest of a specific graph cut called pruning in this context.

\footnotetext{
* This work has been developed in the framework of the project BIGGRAPHTEC2013-43935-R, financed by the Spanish Ministerio de Economía y Competitividad and the European Regional Development Fund (ERDF).
} 
We discuss and evaluate various pruning techniques formulated as the search in the BPT of a partition optimizing a certain criterion. The criteria we analyze take into account the specific nature of PolSAR data and the presence of speckle noise resulting from the coherent integration of the electromagnetic waves. The main contributions of this paper compared to $[2,3,17]$ is the proposal of new pruning strategies for PolSAR images as well as the objective evaluation of the resulting partitions thanks to a set of realistic simulated PolSAR images where the underlying ground truth is available [8].

The paper is organized as follows: Section 2 is a short introduction on PolSAR data. Section 3 discusses the BPT creation and its processing with graph cut. Four pruning criteria useful for segmentation of PolSAR images are presented in section 4 and evaluated in section 5. A preliminary study of the interest of Minkowski norms in the definition of the optimization criterion is presented in section 6. Finally, conclusions are reported in section 7 .

\section{PolSAR data}

Synthetic Aperture Radars (SAR) are active microwave imaging systems. They are becoming increasingly popular for Earth observation because they work independently of the day and night cycle and of weather conditions. A SAR system essentially transmits an electromagnetic wave and records its echo to localize targets. In order to achieve a high spatial resolution, narrow beamwidth or equivalently large antennas are necessary. SAR systems deal with this issue by making use of the relative motion between the sensor and the target. As the radar moves, it repeatedly illuminates the target with electromagnetic pulses. The echoes are coherently recorded and combined in a post-processing that synthesizes a very large array and creates a high resolution image. The speckle noise results from the coherent addition of the scattered electromagnetic waves and is considered as one of the main problems for the exploitation of SAR data.

In the early 90's, multidimensional systems were developed. They provide complex SAR images $\left[S_{1}, S_{2}, \ldots, S_{m}\right]$ by introducing some sort of diversity. An important example is Polarimetric SAR (PolSAR) [5, 13] where the diversity is based on considering different polarization states for the transmitted and received electromagnetic waves. This makes SAR data sensitive to the target geometry, including vegetation, and to the dielectric properties of the target. For every resolution cell, a PolSAR system measures the scattering matrix:

$$
\mathbf{S}=\left[\begin{array}{ll}
S_{h h} & S_{h v} \\
S_{v h} & S_{v v}
\end{array}\right]
$$

where $h$ and $v$ represent the horizontal and vertical polarization states and $S_{p q}$ for $p, q \in\{h, v\}$ denotes the complex SAR image where the reception (transmission) polarization states is $p(q)$.

Since the dimensions of the resolution cell are normally larger that the wavelength of the electromagnetic wave, the scattered wave results from the coherent combination of many waves. This coherent addition process is known as the 
speckle. Although the speckle represents a true electromagnetic measurement, its complexity is such that it is considered as a random process. Rewriting the $\mathbf{S}$ matrix as a vector $\mathbf{k}[6]$ :

$$
\mathbf{k}=\left[S_{h h}, \sqrt{2} S_{h v}, S_{v v}\right]^{T}
$$

$\mathbf{k}$ is characterized by a three dimensional zero-mean complex Gaussian pdf:

$$
p_{\mathbf{k}}(\mathbf{k})=\frac{1}{\pi^{3}|\mathbf{C}|} \exp \left(-\mathbf{k}^{H} \mathbf{C}^{-1} \mathbf{k}\right)
$$

Therefore, the distribution of $\mathbf{k}$ is completely described by the Hermitian positive definite covariance matrix:

$$
\begin{aligned}
\mathbf{C} & =E\left\{\mathbf{k k}^{H}\right\} \\
& =\left[\begin{array}{ccc}
E\left\{S_{h h} S_{h h}^{H}\right\} & \sqrt{2} E\left\{S_{h h} S_{h v}^{H}\right\} & E\left\{S_{h h} S_{v v}^{H}\right\} \\
\sqrt{2} E\left\{S_{h v} S_{h h}^{H}\right\} & 2 E\left\{S_{h v} S_{h v}^{H}\right\} & \sqrt{2} E\left\{S_{h v} S_{v v}^{H}\right\} \\
E\left\{S_{v v} S_{h h}^{H}\right\} & \sqrt{2} E\left\{S_{v v} S_{h v}^{H}\right\} & E\left\{S_{v v} S_{v v}^{H}\right\}
\end{array}\right]
\end{aligned}
$$

where $E\{x\}$ is the statistical expectation of $x$. The Maximum Likelihood Estimation (MLE) of $\mathbf{C}$, i.e., the multilook, under the assumption of statistical ergodicity and homogeneity, is obtained by substituting the statistical expectation by an averaging:

$$
\mathbf{Z}=\left\langle\mathbf{k k}^{H}\right\rangle_{n}=\frac{1}{n} \sum_{i=1}^{n} \mathbf{k}_{i} \mathbf{k}_{i}^{H}
$$

where $n$ indicates the number of independent looks or samples employed to estimate $\mathbf{C}$ and $\mathbf{k}_{i}$ is the $i^{\text {th }}$ sample vector. The estimated covariance matrix $\mathbf{Z}$ is statistically characterized by a Wishart distribution [12].

\section{BPT creation and processing through graph cut}

The BPT creation starts by the definition of an initial partition which can be composed of individual pixels as in $[2,3]$. While this strategy guarantees a high precision as starting point of the merging process, it also implies high computational and memory costs as many regions have to be handled. As an alternative, the initial partition may correspond to an over-segmentation as a super-pixel partition. This initial partition issue was studied in [17] where several alternative strategies were evaluated. The main conclusion of this study is that the use of super-pixel partition as initial partition of the merging process can indeed drastically reduce the computational load of the BPT creation without any significant impact on the quality of the regions and partitions represented by the tree. One of the key point however is to use a denoising filter adapted to PolSAR images such as $[14,7]$ before computing the super-pixel partitions. The best combination found in [17] involves the use of the $\sigma$-Lee denoising filter [14] followed by the SLIC algorithm [1] to compute the super-pixel. Only the three 


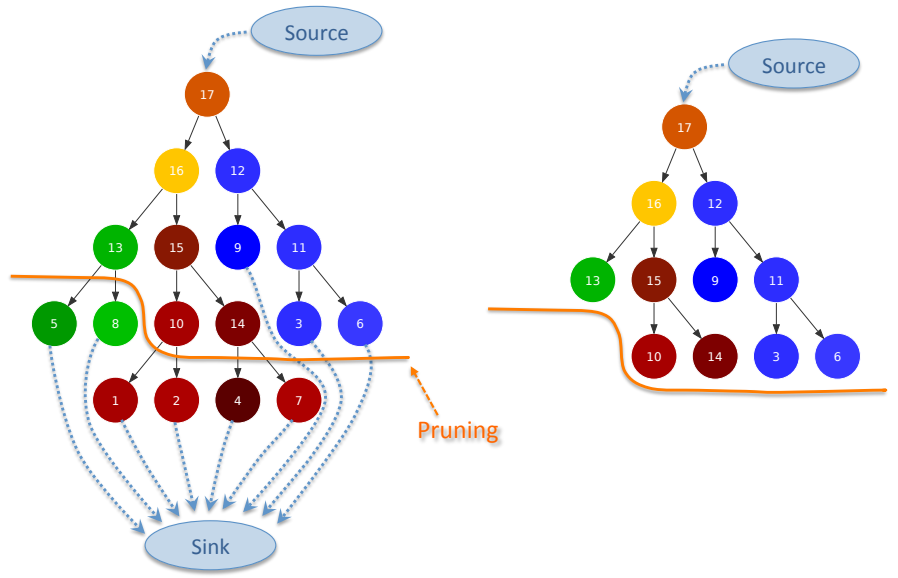

Fig. 1. Illustration of the pruning on a BPT. Left: The root of the BPT is connected to a source node and the leaves are connected to a sink node. The pruning creates two connect component where siblings belong to the same connected component. Right: the pruned BPT is the connected component that includes the source. Its leaves define the extracted partition.

diagonal elements of the covariance matrices $\mathbf{Z}$ (after denoising) have been used to generate the super-pixels.

Once the initial partition is defined, the BPT construction is done by iteratively merging the pair of most similar neighboring regions. In the PolSAR case of interest here, the information carried by pixels $(i, j)$ of an image $I$ corresponds to the covariance matrix $\mathbf{Z}_{i j}^{I}$ after denoising. To construct the BPT, we need to define a region model and a similarity measure between neighboring regions.

We use the strategy defined in [2] where regions $R$ are modeled by their mean covariance matrix $\mathbf{Z}_{R}=\frac{1}{|R|} \sum_{i, j \in R} \mathbf{Z}_{i j}^{I}$, where $|R|$ is the region number of pixels. If the region is homogeneous, this estimation corresponds to the MLE defined by Eq. 5. The distance between neighboring regions, which defines the merging order, relies on the Symmetric revised Wishart dissimilarity [2]. The revised Wishart dissimilarity measure [9] is based on a statistical test assuming that the two regions follow a Wishart pdf and that one pdf is known. Thus, it is not symmetric as it depends on which region pdf is assumed to be known. In order to define the merging order, a symmetric version is used:

$$
d_{R W}\left(R_{1}, R_{2}\right)=\left(\operatorname{tr}\left(\mathbf{Z}_{R_{1}}^{-1} \mathbf{Z}_{R_{2}}\right)+\operatorname{tr}\left(\mathbf{Z}_{R_{2}}^{-1} \mathbf{Z}_{R_{1}}\right)\right)\left(\left|R_{1}\right|+\left|R_{2}\right|\right)
$$

where $R_{1}, R_{2}$ are the two neighboring regions and $\operatorname{tr}(\mathbf{A})$ denotes the trace of the $\mathbf{A}$ matrix and $\mathbf{A}^{-1}$ its inverse.

Once the BPT has been constructed, it can be used for a wide range of applications including filtering, segmentation or classification. In many cases, the application relies on the extraction of a partition from the BPT. This process can be seen as a particular graph cut called pruning that can be formally defined as 
follows: Assume the tree root is connected to a source node and that all the tree leaves are connected to a sink node. A pruning is a graph cut that separates the tree into two connected components, one connected to the source and the other to the sink, in such a way that any pair of siblings falls in the same connected component. The connected component that includes the root node is itself a BPT and its leaves define a partition of the space. This process is illustrated in Fig. 1. In the sequel, we discuss several examples of PolSAR image pruning.

\section{Optimum pruning of BPTs for PolSAR data}

As previously mentioned, the extraction of a partition from the BPT can be defined by a pruning strategy. In this paper, we are interested in pruning techniques that extract partitions optimizing a certain criterion. More precisely, we restrict ourselves to additive criteria, that are criteria defined as:

$$
C=\sum_{R} \phi_{R}
$$

where $R$ is a set of regions described in the BPT that forms a partition and $\phi_{R}$ a measure depending on $R$.

This type of criterion can be efficiently minimized using an dynamic programing algorithm originally proposed in [18] for global optimization on BPT. The solution consists in propagating local decisions in a bottom-up fashion. The BPT leaves are initially assumed to belong to the optimum partition. Then, one checks if it is better to represent the area covered by two sibling nodes as two independent regions $\left\{R_{1}, R_{2}\right\}$ or as a single region $R$ (the common parent node of $R_{1}$ and $R_{2}$ ). The selection of the best choice is done by comparing the criterion $\phi_{R}$ evaluated on $R$ with the sum of the criterion values $\phi_{R_{1}}$ and $\phi_{R_{2}}$ :

$$
\text { If } \phi_{R} \leq \phi_{R_{1}}+\phi_{R_{2}} \begin{cases}\text { then } & \text { select } R \\ \text { else } & \text { select } R_{1} \text { and } R_{2}\end{cases}
$$

The best choice (either " $R$ " or " $R_{1}$ plus $R_{2}$ ") is stored in the node representing $R$ with the corresponding criterion value $\left(\phi_{R}\right.$ or $\left.\phi_{R_{1}}+\phi_{R_{2}}\right)$. The procedure is iterated up to the root and defines the best partition. This algorithm finds the global optimum of the criterion on the tree and the selected regions form a partition of the image.

We discuss now four pruning techniques for low-level processing and grouping of PolSAR data. The main goal of theses pruning techniques is to segment the images so that a precise estimation of the region contours as well as of the polarimetric parameters can be done.

The first and most obvious pruning technique relies on the adaptation of Square Error (SE) to the matrix case, here the covariance matrices. It simply consists in computing the matrix norm of the difference between the covariance matrices $\mathbf{Z}_{i j}^{I}$ of the pixels belonging to a given region $R$ and the covariance 
matrix presenting the region model $\mathbf{Z}_{\mathbf{R}}$ :

$$
\phi_{R}=\sum_{i, j \in R}\left\|\mathbf{Z}_{i j}^{I}-\mathbf{Z}_{R}\right\|_{F}
$$

where $\|$.$\| represents the so-called Frobenius norm { }^{1}$. This criterion essentially enforces the homogeneity of regions. However, on its own, it is useless because a partition made of the initial leaves of the BPT will be optimum as this is where the deviation of the individual pixels with respect to the region mean will be minimized. Following classical approaches in functional optimization, $\phi_{R}$ can be interpreted as a data fidelity term and combined with a data regularization term which encourages the optimization to find partitions with a reduced number of regions. As simple data regularization, we use a constant value $\lambda$ that penalizes the region presence. Therefore, the final homogeneity-based criterion to be minimized is given by $C=\sum_{R} \phi_{R}$ with $\phi_{R}$ defined as follows:

$$
\phi_{R}^{S E}=\sum_{i, j \in R}\left\|\mathbf{Z}_{i j}^{I}-\mathbf{Z}_{R}\right\|_{F}+\lambda
$$

This first pruning criterion may be interesting to extract homogenous regions in terms of the data covariance matrix but it does not take into account the presence of the speckle noise. As discussed above the speckle noise is a random process that is complex to characterize but it is often approximated by a multiplicative noise [6]. Therefore, a second pruning criterion can be derived from the first one normalizing the homogeneity measure by the average norm of the region model. The corresponding $\phi_{R}$ can be written as:

$$
\phi_{R}^{S A R \_S E}=\sum_{i, j \in R}\left\|\mathbf{Z}_{i j}^{I}-\mathbf{Z}_{R}\right\|_{F} /\left\|\mathbf{Z}_{R}\right\|_{F}+\lambda
$$

The third pruning criterion relies on the region similarity measure used for the construction of the BPT. In section 3, we mentioned that a Wishart-based measure was used to compute the similarity between neighboring regions. Eq. 6 can be adapted to measure the similarity between pixels and the region model. It would lead to an expression such as: $\operatorname{tr}\left(\left(\mathbf{Z}_{i, j}^{I}\right)^{-1} \mathbf{Z}_{R}\right)+\operatorname{tr}\left(\left(\mathbf{Z}_{R}\right)^{-1} \mathbf{Z}_{i, j}\right)$. However, the matrix inversion at the pixel level is computationally demanding and the matrix may even be singular. Therefore, we used a simplified formulation of this measure by taking into account only the diagonal elements of the matrices.

$$
\phi_{R}^{\text {Wishart }}=\sum_{i, j \in R} \sqrt{\sum_{k=1,2,3}\left(\frac{\mathbf{Z}_{i j}^{I}(k, k)^{2}+\mathbf{Z}_{R}(k, k)^{2}}{\mathbf{Z}_{i j}^{I}(k, k) \mathbf{Z}_{R}(k, k)}\right)}+\lambda
$$

where $\mathbf{Z}_{i j}^{I}(k, k)$ and $\mathbf{Z}_{R}(k, k)$ respectively represent the diagonal elements of covariance matrices $\mathbf{Z}_{i j}^{I}$ and $\mathbf{Z}_{R}$.

\footnotetext{
${ }^{1}$ The Frobenius norm of matrix $A$ with elements $[a(k, l)]$ is: $\|A\|_{F}=\sqrt{\sum_{k, l} a(k, l)^{2}}$.
} 
Finally, the last pruning criterion relies on a geodesic distance adapted to the cone of positive definite Hermitian matrices [4]. This measure exploits the geometry of the space defined by the covariance matrices and is given by: $\left\|\log \left(\mathbf{Z}_{R}^{-1 / 2} \mathbf{Z}_{i, j}^{I} \mathbf{Z}_{R}^{-1 / 2}\right)\right\|_{F}$, where $\log ($.$) represents the matrix logarithm. As pre-$ viously, since this measure is quite complex to compute, we use a simplified version taking into account only the diagonal elements of the matrices. The fourth pruning criterion is then given by:

$$
\phi_{R}^{\text {Geodesic }}=\sum_{i, j \in R} \sqrt{\sum_{k=1,2,3} \ln ^{2}\left(\frac{\mathbf{Z}_{i j}^{I}(k, k)}{\mathbf{Z}_{R}(k, k)}\right)}+\lambda
$$

where $\ln$ represents the natural logarithm.

\section{Evaluation}

To objectively measure the performances of the pruning discussed in the previous section, we rely on a dataset of PolSAR images on which the ground-truth polarimetric information is available. More precisely, we use the set of simulated PolSAR images [8] where the underlying ground-truth, i.e. the class regions, is modeled by Markov Random Fields. A set of typical polarimetric responses has been extracted from an AIRSAR image (L-band) so that they represent the 8 classes found in the $H / \bar{\alpha}$ plane and randomly assigned to each class. Then, single look complex images have been generated from the polarimetric responses using a Cholesky decomposition [11]. Examples of images and their corresponding ground-truth, denoised images and super-pixel partitions are presented in Fig. 2.

Thanks to this dataset with ground-truth, we can measure the quality of the pruning techniques in the context of segmentation because we know the ideal partition. Fig. 3 shows the evaluation of the segmentation results as classically done in the supervised case through Precision and Recall curves. On the left side, the so-called Precision and Recall for boundaries [15] is presented. In this case, each partition is evaluated by considering all pairs of neighboring pixels and by classifying them in either boundary or interior segments. The Precision and the Recall values of this classification are evaluated by comparison with the classification resulting from the ground-truth partition. In addition to this boundary-oriented evaluation, a region-oriented evaluation known as the Precision and Recall for objects and parts [16] is presented on the right side of Fig. 3. In this context, regions of the partition are considered as potential candidates to form regions of the ground-truth partition, and are classified as correct or not. In both cases, the curves are formed by modifying the $\lambda$ value to get coarser or finer partitions. The ideal system has Precision and Recall values equal to one.

As can be seen in Fig. 3, the region-oriented evaluation is more severe than the boundary-oriented evaluation. This is to be expected as the boundary measure simply checks whether boundary elements in the ground-truth partitions match boundary elements of the partitions extracted from the BPT. As the 

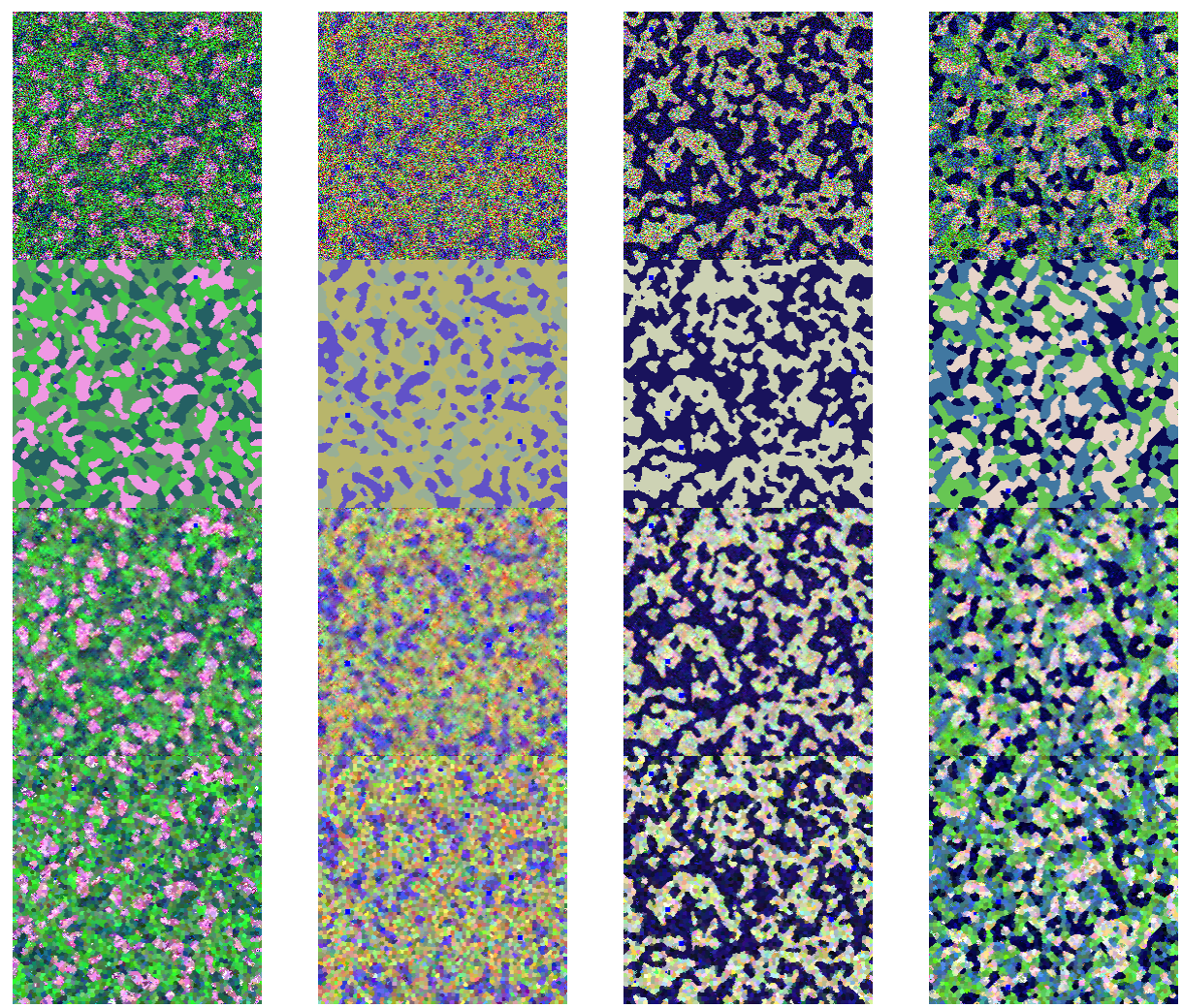

Fig. 2. Examples of original PolSAR images (first row), their corresponding groundtruth (second row), denoised images (third row) and super-pixel partitions (fourth row). RGB-pauli color coding: the polarimetric channels $|H H-V V|,|H V|$ and $|H H+V V|$ are assigned to the RGB channels respectively.

partitions are rather dense, it not very difficult to find matching boundary elements. However, the boundary measure does not actually analyze whether the ground-truth regions correspond to regions of the partitions computed from the BPT. This issue is evaluated by the "objects and parts" measure (see [16] for details on this issue). However, the conclusions on both plots are the same: the best pruning technique is the one based on $\phi_{R}^{S A R-S E}$ (Eq. 11). The pruning techniques based on the Wishart pdf (Eq. 12) and the geodesic distance (Eq. 13) seems to provide interesting results for very coarse partitions (precision values close to one obtained for high values of $\lambda$ ).

Precision and Recall curves describe the performances for the complete range of pruning parameter values. However, they do not efficiently describe the system sensitivity to the parameter value. To this end, Fig. 4 presents the $F$ value as a function of the pruning parameter. The $F$ value is classically used to summarize the Precision $P$ and Recall $R$ trade-off. It is the harmonic mean of $P$ and $R$ : 

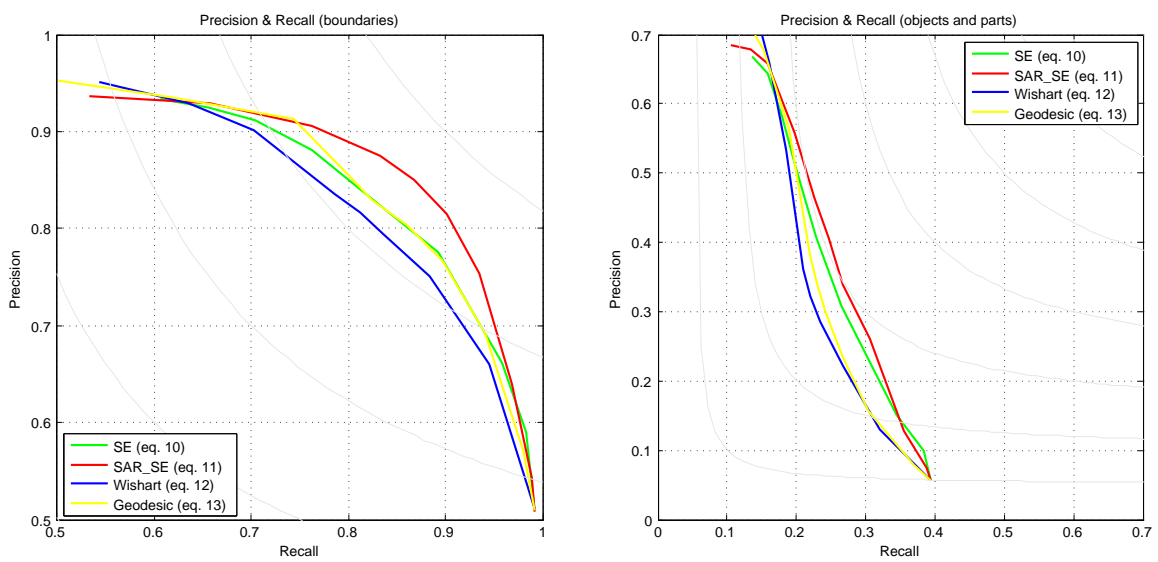

Fig. 3. Precision and Recall (PR) performances of the four pruning techniques (average over the entire dataset). Left: PR for boundaries, Right: PR for objects and parts.
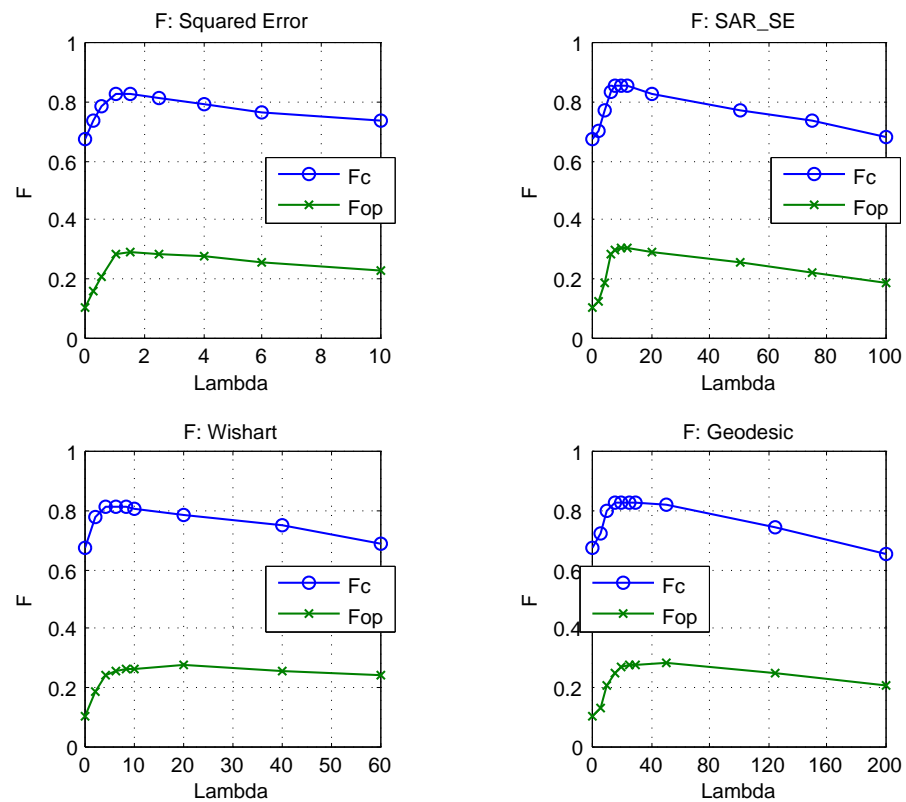

Fig. 4. $F$ value as a function of the pruning parameter. $F_{b}\left(F_{o p}\right)$ corresponds to the Precision and Recall for boundaries (object and parts) curves. Top: $\phi_{R}^{S E}$ and $\phi_{R}^{S A R} R_{-} E$, Bottom: $\phi_{R}^{\text {Wishart }}$ and $\phi_{R}^{G e o d e s i c}$.

$F=2 P R /(P+R)$. Fig. 4 shows that all pruning techniques provide stable results for a wide range of $\lambda$ values. If we consider the best pruning approach $\phi_{R}^{S A R \_S E}$ for example, this means that, in practice, $\lambda$ values between 5 and 15 will extract similar partitions and there is no need to fine tune the parameter. 

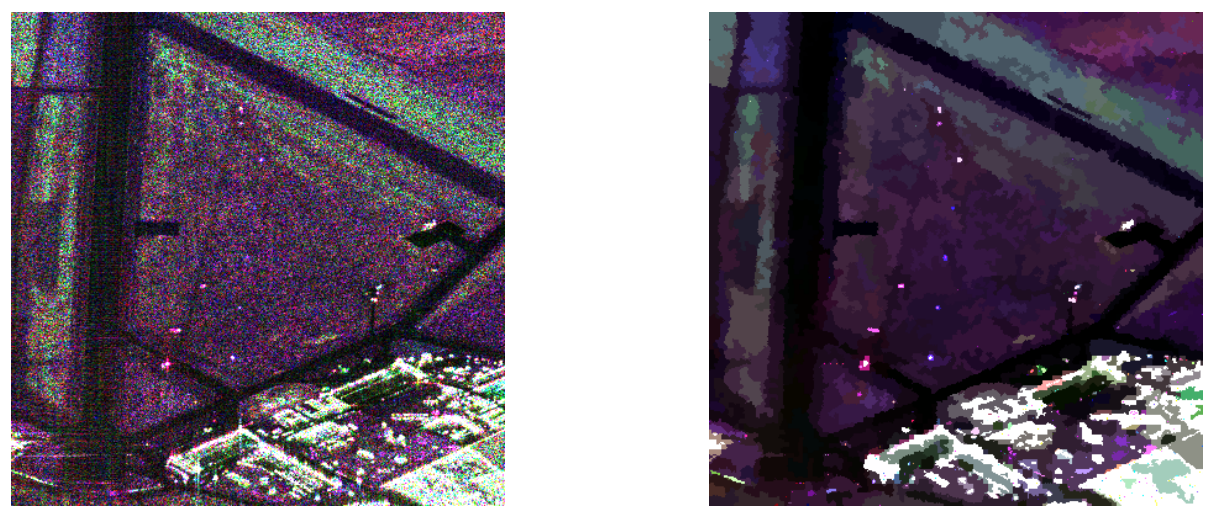

Fig. 5. Results on real images. Right: Original images (RGB Pauli composition). Left: Pruning with $\phi_{R}^{S A R_{-} S E}$ (Eq. 11).
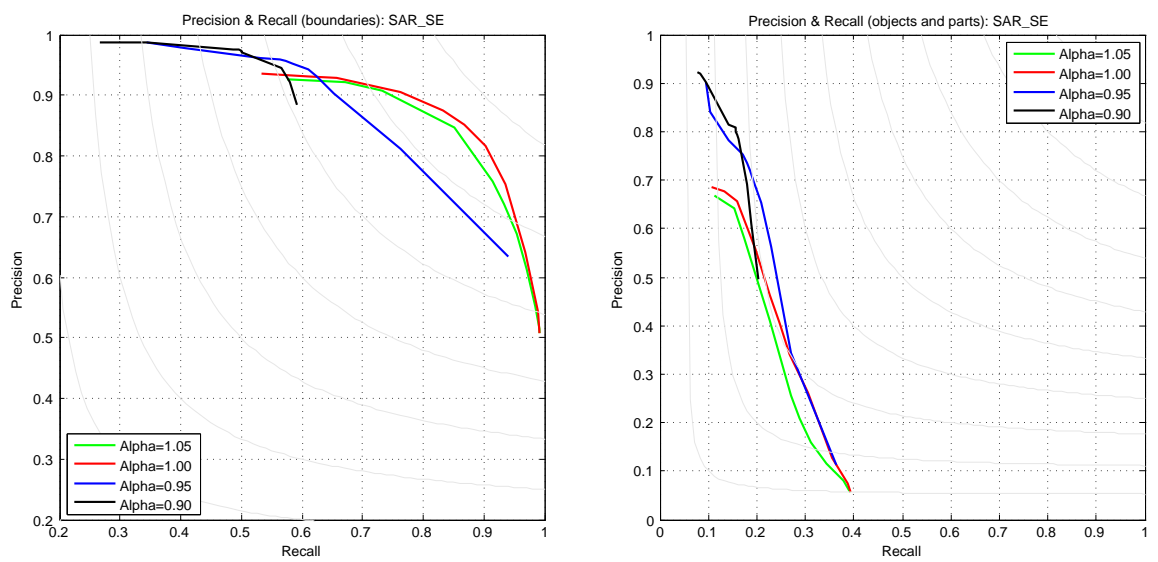

Fig. 6. Precision and Recall (PR) performances of the h-increasing pruning technique defined by Eq. 14 (average over the entire dataset). Left: PR for boundaries, Right: PR for objects and parts.

Finally, the pruning with $\phi_{R}^{S A R_{-} S E}$ (Eq. 11) has been used on a L-band fully polarimetric data set acquired in 2003 by the Deutsches Zentrum für Luftund Raumfahrt (DLR) ESAR system over the area of the Oberpfaffenhofen airport near Munich, Germany. The images are Single Look Complex with a pixel size of $1,5 \times 1,5 \mathrm{~m}$. Results are shown in Fig. 5 together with the original image. They visually highlight the interest of the BPT to perform low-level processing of PolSAR images while preserving the spatial resolution of the content.

\section{Potential on non-additive criteria}

It has been recently shown that the algorithm identifying optimum partitions as described in section 4 is valid for a larger class of criteria than purely ad- 
ditive criteria such as $C=\sum_{R} \phi_{R}$. In particular [19] introduces the notion of h-increasing criterion which is sufficient (and necessary) to guarantee that the search algorithm identifies the optimum partition. Moreover, [10] shows that Minkowski norms: $C=\left(\sum_{R} \phi_{R}^{\alpha}\right)^{\frac{1}{\alpha}}, \forall \alpha$, are h-increasing as well as their combination by addition or supremum. Therefore the same search algorithm can be used for this larger class of criteria.

As a preliminary experiment to study the potential of h-increasing criteria beyond additive ones $(\alpha=1)$ for PolSAR data, we have studied a simple modification of the best pruning identified in the previous section: $\phi_{R}^{S A R \_S E}$. We evaluated the performances of the pruning based on the following criterion:

$$
C=\sum_{R}\left(\sum_{i, j \in R}\left(\left\|\mathbf{Z}_{i j}^{I}-\mathbf{Z}_{R}\right\|_{F} /\left\|\mathbf{Z}_{R}\right\|_{F}\right)^{\alpha}\right)^{\frac{1}{\alpha}}+\lambda
$$

The results in terms of precision and recall are shown in Fig. 6 for four values of the $\alpha$ parameter. As can be seen, the classical additive approach $(\alpha=1)$ gives the best results in the sense that it allows to get closer to the ideal point of Precision=1 and Recall=1. However, $\alpha$ values lower than one seem to give better results for coarse partitions (large $\lambda$ values implying a cut close to the root node). This is an interesting result that suggests that the use of Minkowski norms has to be further investigated at least for PolSAR data.

\section{Conclusions}

This paper has discussed the interest of Binary Partition Trees (BPTs) for PolSAR images and highlighted the usefulness of a particular type of graph cut called pruning to extract partitions from the BPT. Four specific pruning techniques involving the global optimization of a criterion related the region homogeneity have been evaluated. The best pruning strategy relies on a normalized version of squared error where the normalization takes into account the specific multiplicative nature of the speckle noise. Finally, preliminary results suggest that the use of Minkowski norms has to be further investigated as it proved to provide good results for pruning close to the root of the tree.

\section{References}

1. R. Achanta, A. Shaji, K. Smith, A. Lucchi, P. Fua, and S. Süsstrunk. SLIC superpixels compared to state-of-the-art superpixel methods. IEEE Trans. on Pattern Analysis and Machine Intelligence, 34(11):2274 - 2282, 2012.

2. A. Alonso-Gonzalez, C. Lopez-Martinez, and P. Salembier. Filtering and segmentation of polarimetric SAR data based on binary partition trees. IEEE Trans. on Geoscience and Remote Sensing, 50(2):593-605, 2012.

3. A. Alonso-Gonzalez, S. Valero, J. Chanussot, C. Lopez-Martinez, and P. Salembier. Processing multidimensional SAR and hyperspectral images with binary partition tree. Proceedings of IEEE, 101(3):723-747, 2013. 
4. F. Barbaresco. Interactions between symmetric cone and information geometries: Bruhat-tits and siegel spaces models for high resolution autoregressive doppler imagery. In Emerging Trends in Visual Computing, volume 5416, pages 124-163. LNCS, 2009.

5. S. Cloude. Polarisation Applications in Remote Sensing. Oxford Univ. Press, 2009.

6. S. Cloude and E. Pottier. A review of target decomposition theorems in radar polarimetry. IEEE Trans. on Geosc. and Remote Sens., 34(2):498-518, 1996.

7. C.A. Deledalle, F. Tupin, and L. Denis. Polarimetric SAR estimation based on nonlocal means. In IEEE International Geoscience and Remote Sensing Symposium, IGARSS 2010, 2010.

8. S. Foucher and C. Lopez-Martinez. Analysis, evaluation, and comparison of polarimetric SAR speckle filtering techniques. IEEE Trans. on Image Processing, 23(4):1751-1764, 2014.

9. P. R. Kersten, J.-S. Lee, and T. L. Ainsworth. Unsupervised classification of polarimetric synthetic aperture radar images using fuzzy clustering and EM clustering. IEEE Trans. on Geoscience and Remote Sensing, 43(3):519-527, Mar. 2005.

10. B. R. Kiran. Energetic-Lattice based optimization. PhD thesis, Université ParisEst, 2014.

11. J.-S. Lee, T.L. Ainsworth, J.P. Kelly, and C. López-Martínez. Evaluation and bias removal of multilook effect on entropy/alpha/anisotropy in polarimetric SAR decomposition. IEEE Trans. on Geosc. and Remote Sens., 46(10):3039-3051, 2008.

12. J.-S. Lee, K. Hoppel, S. Mango, and A. Miller. Intensity and phase statistics of multilook polarimetric and interferometric SAR imagery. IEEE Trans. on Geoscience and Remote Sensing, 32(5):1017-1028, Sept. 1994.

13. J.-S. Lee and E. Pottier. Polarimetric Radar Imaging: From Basics to Applications. Boca Raton, FL: CRC Press, 2009.

14. J.-S. Lee, J.H . Wen, T.L. Ainsworth, K.S. Chen, and A.J. Chen. Improved sigma filter for speckle filtering of SAR imagery. IEEE Trans. on Geoscience and Remote Sens., 47(1):202 - 213, 2009.

15. D. Martin, C. Fowlkes, and J. Malik. Learning to detect natural image boundaries using local brightness, color, and texture cues. IEEE Trans. on Pattern Analysis and Machine Intelligence, 26(5):530-549, 2004.

16. J. Pont-Tuset and F. Marques. Measures and meta-measures for the supervised evaluation of image segmentation. In Computer Vision and Pattern Recognition (CVPR), 2013.

17. P. Salembier, S. Foucher, and C. Lopez-Martinez. Low-level processing of PolSAR images with binary partition trees. In IEEE International Geoscience and Remote Sensing Symposium, IGARSS 2014, Quebec, Canada, July 2014.

18. P. Salembier and L. Garrido. Binary partition tree as an efficient representation for image processing, segmentation, and information retrieval. IEEE Trans. on Image Processing, 9(4):561 -576, 2000.

19. J. Serra. Hierarchy and optima. In Debled-Renneson et al., editor, Discrete Geometry for Computer Imagery, LNCS 6007, pages 35-46. Springer, 2011.

20. S. Valero, P. Salembier, and J. Chanussot. Hyperspectral image representation and processing with binary partition trees. IEEE Trans. on Image Processing, 22(4):1430 - 1443, 2013.

21. M.A. Veganzones, G. Tochon, M. Dalla-Mura, A.J. Plaza, and J. Chanussot. Hyperspectral image segmentation using a new spectral unmixing-based binary partition tree representation. IEEE Trans. on Image Proc., 23(8):3574 - 3589, 2014. 